\title{
A comparative study of efficacy and safety of oral ketamine and oral midazolam as premedicant for paediatric cardiac catheterization
}

\author{
Soma Ganesh Raja ${ }^{1, *}$, Gowri Shankar Sarate ${ }^{2}$ \\ ${ }^{\mathbf{1}}$ Assistant Professor, Dept. of Anaesthesia, Sri Ramachandra Medical College and Research Institute, Chennai, Tamil Nadu, \\ ${ }^{2}$ Professor, Dept. of Anaesthesia, SMBT Institute of Medical Sciences \& Research Centre, Nashik, Maharashtra, India \\ *Corresponding Author: \\ Email: drsoms@gmail.com
}

Received: $12^{\text {th }}$ December, 2017

Accepted: $25^{\text {th }}$ January, 2018

\begin{abstract}
Use of an effective sedative premedicant significantly minimizes the emotional trauma associated with perioperative anxiety and its sequelae. Currently, oral midazolam is the most commonly used premedicant in most of the countries followed by oral ketamine.

Aims and Objectives: To compare the efficacy and safety of oral ketamine and oral midazolam as premedicant for paediatric cardiac catheterization.

Materials and Methods: This study was carried out in the tertiary care centre in sixty patients randomly assigned into two groups of thirty patients each, to compare the efficacy of oral ketamine and oral midazolam pre medication as sedative for paediatric patients undergoing cardiac catheterization using various parametes and observation scale of response and Visual Analogue scale. Data collected was analysed and the statistical significance of these intra-group and inter-group observations thus made was determined using either a 'Chi Square test' or an 'Unpaired T-test' or Mann whitney test.

Results: The demographic trends in all the groups were comparable. The type of procedure performed were also comparable in both the groups. The mean heart rate, mean systolic and diastolic blood pressures, mean arterial blood pressure were higher in the ketamine group than in the midazolam group. Though there was fall in oxygen saturation in both the groups the ketamine group required oxygen supplementation more than midazolam group though it was statistically insignificant.

The mean onset of sedation was earlier for midazolam than for ketamine. Also the parental separation was smoother and calmer in patients who had oral midazolam than oral ketamine.

Similarly while positioning the children for cath study also patients medicated with midazolam showed better response than those medicated with ketamine. The response to local anaesthetic injection during femoral vessels cannulation also showed midazolam with better results. The adverse effects such as secretions, nystagmus, nausea, vomiting, delirium were more common with ketamine. The mean time spent in recovery was 53.33 minutes with ketamine group and also parental satisfaction in terms of VAS score is better and favours midazolam than ketamine.

Conclusions: Oral midazolam is a better premedication of choice than ketamine for paediatric patients undergoing cardiac catheterization.
\end{abstract}

Keywords: Sedation, Anxiety, Procedure.

\section{Introduction}

Depending upon the age, developmental maturity and past surgical experiences, children suffer from varying degrees of terror while facing the prospects of any surgical procedure. Almost $50 \%$ of children undergoing surgery become anxious in the perioperative period. They are principally worried about pain and separation from their parents. ${ }^{1}$ The risk factors which seem to be associated with high incidence of perioperative anxiety in children include: Age one to six years, shy and inhibited nature, previous poor quality medical encounters, poor social adaptability and increased parental anxiety. ${ }^{2,3}$

The role of an anaesthesiologist in preparing children for surgical procedures is to minimize potentially adverse psychological and physiological effects of anaesthetic techniques. Children who are premedicated have shown a lower incidence of maladaptive postoperative behavioural changes. ${ }^{4}$ Thus there are several compelling reasons to treat children's anxiety preoperatively. ${ }^{4-7}$
Cardiac catheterization in paediatric population is on increasing trend due to the diagnostic and therapeutic advances in cardiology especially congenital heart diseases. More frequently anaesthetists are being called upon to provide support in sedating, anaesthetizing or and resuscitating these patients. To avoid emotional trauma associated with parent-child separation and facemask application during oxygen supplementation, it was planned to premedicate the children, appearing for elective diagnostic cardiac catheterisation, with the most commonly utilized premedicants namely ketamine and midazolam via the oral route.

The purpose of this study is to compare the efficacy of oral ketamine and oral midazolam as pre medication for diagnostic cardiac catheterization.

\section{Materials and Methods}

This study was conducted in the Tertiary care centre, Sir J J Group of Government Hospitals, attached to Grant Medical College, Mumbai. After getting 
Ethical committee clearance for conducting this study, the study was conducted among sixty paediatric patients who were posted for elective cardiac catheterization. The patients were randomly allocated into two groups.

Group A - 30 patients - Received $6 \mathrm{mg} / \mathrm{kg}$ of ketamine mixed with $25 \%$ Dextrose volume adjusted to age.

Group B - 30 patients - Received $0.6 \mathrm{mg} / \mathrm{kg}$ of midazolam mixed with $25 \%$ Dextrose volume adjusted to age.

Patients who were posted for elective diagnostic cardiac catheterization between 5 to $15 \mathrm{yrs}$ of age of both the gender were included,

Emergency cases and patients of ASA [American society of Anaesthesiologist] Grade IV and above, children with known allergy to ketamine or midazolam, children with Atrial fibrillation, congestive cardiac failure, left ventricular failure, extreme tachycardia etc. children with high pulmonary artery pressures (more than $40 \mathrm{~mm} \mathrm{Hg}$ ), children with respiratory system diseases such as upper/ lower respiratory tract infections, bronchial asthma, chronic bronchitis etc., Children with central nervous system dysfunction such as seizure disorders, increased intracranial pressure, neuropsychiatric diseases etc.. Children with increased intra ocular pressure such as glaucoma, children with known allergy to local anaesthetics, children with prolonged therapy with enzyme inducing drugs. Children who refuse to take whole dose of oral medications were excluded.

\section{Materials}

1. Oral Midazolam: Injection Midazolam hydrochloride - available as $1 \mathrm{ml}$ ampoule containing $5 \mathrm{mg}$. The brand used was Mezolam. It is preservative free. It is diluted with $25 \%$ dextrose to give to patients at a dose of $0.6 \mathrm{mg} / \mathrm{kg}$ volume adjusted to age.

2. Oral Ketamine: Injection ketamine hydrochloride -available as $2 \mathrm{ml}$ ampoule containing $100 \mathrm{mg}$. The brand used was Ketalar. It is preservative free. It is diluted with $25 \%$ dextrose to give patients at a dose of $6 \mathrm{mg} / \mathrm{kg}$ volume adjusted to age.

\section{Methods}

\section{Prior to the day of cardiac catheterization}

Pre-anesthetic check-up was carried out in all patients and they were optimized for the surgery.

The following investigations were carried out in all patients:

1. Complete hemogram

2. Random blood sugar

3. Serum electrolytes

4. Liver function tests

5. Renal function tests

6. Coagulation profile

7. Chest radiograph

8. Electro-cardiogram

9. 2 D Echo

Patients/parents/guardian were explained the anesthetic technique and the necessity of general anesthesia if the situation warrants and the risks and complications associated with it and an informed valid written consent was taken.

\section{On the day of catheterization study}

1. The attending anesthesiologist checked personally all the resuscitation equipments and drugs before the starting of procedure necessary for the patient.

2. Patients' 'nil by mouth' status was confirmed prior to surgery.

3. The children along with their parents were taken to the pre medication area.

4. Baseline vital parameters such as heart rate, Blood pressure, saturation in pulse oximetry were measured and noted.

5. The children were given the premedication orally as per the group and were motivated to take the drug fully in a single time.

6. The children were observed for their heart rates, saturation from pulseoximeter, Blood pressure, respiratory rate, at $0,1,5,10,15,24,35$ minutes after giving the oral drug.

7. An observational scale of patient's response was assessed as per the following table.

Table 1: Observational scale of response

\begin{tabular}{|l|c|}
\hline \multicolumn{1}{|c|}{ Scale } & Patient response \\
\hline Marked response (A) & Marked response or distress \\
& Extreme agitation \\
& Physical resistance /purposeful movements \\
Prolonged crying
\end{tabular}


1. All the patients were closely observed and monitored. The time at which observable onset of sleep / sedation occurs were noted for all patients. All vital signs were noted simultaneously.

2. After 30 minutes of giving oral premedication, $1 \%$ lignocaine were injected for intravenous cannula placement. Patient response to local anesthetic were assessed by using the observational scale of marked response versus moderate response versus minimal response.

3. After securing intravenous access, the children were transported to the cardiac catheterization lab.

4. They were separated from their parents and moved to the cardiac catheterization table where they were positioned, prepared and draped.

5. 1\% lignocaine were infiltrated subcutaneously, over the femoral vessels to allow cannulation.

6. At separation. Positioning and at cannulation, patient response were assessed once again by the three tier observational scale of responses.

7. At positioning and cannulation and for the reminder of the procedure, the attending anaesthesiologist would monitor all vital signs and administer additional sedatives based on patient responses and if required.

8. The additional medication will be propofol $0.5 \mathrm{mg}$ per kg over boluses.

9. The patients were observed for the cardio respiratory effects of propofol and any change in heart rate or fall in saturation as per pulse oximeter were recorded.

10. A fall in saturation of more than six point change was supplemented with oxygen 4 litres per minute via face mask.

11. Doses of propofol were tracked and when more than ten boluses were used an infusion of 100 microgram per $\mathrm{kg}$ per minute was started.

III. Post catheterization study

1. Once the procedure is over, the children are transported to the recovery area

2. Time spent in recovery was noted.

3. The routine hospital recovery discharge criteria was followed and includes

4. All patients were awake and back to baseline level of consciousness.

5. All protective reflexes were intact.

6. Oxygen saturation was back to pre-sedation level.

7. Pain was controlled and a prescription for supplementary pain medication was given if indicated.

8. The IV cannula was only removed when there were no signs of nausea.

9. All vital signs were stable.

10. Patients were asked if they wanted a little water or cool drink, to see if they could swallow and keep it in. (optional)
11. The child was able to sit unattended, speak and answer an age appropriate question before discharge.

12. The child was then handed over to a responsible person usually the parent and sent to the ward.

13. After four hours, a final interview was conducted with the patient and parents.

14. The child was asked about recall of the infiltration of local anesthetic for intravenous cannula or for femoral cannulation or any other events in the catheterization laboratory. Any positive response was judged as failure to provide amnesia.

15. The parents completed a visual analogue scale as a measure of satisfaction with sedation. A $10 \mathrm{~cm}$ unmarked line labeled failed sedation at the left end (0) and excellent sedation at right end (10) was presented parents marked their assessment and these marks will later be given a numeric value by ruler measurement.

16. The parents will also be asked about adverse effects such as nausea or any complications.

\section{Statistical Analysis}

The statistical significance of these intra-group and inter-group observations thus made was determined using either a 'Chi Square test' or an 'Unpaired T-test'. The result if proved to be significant, the level of significance was statistically shown by using P-value, where a P-value of $<0.05$ was considered to be significant.

\section{Results}

The mean age of children in ketamine group is 9.3yrs (+/- 2.38yrs) where as it is $9.23 \mathrm{yrs}(+/-2.24 \mathrm{yrs})$ which is not statistically significant. The mean weight of children in ketamine group is $21.17 \mathrm{~kg}(+/-4.65 \mathrm{~kg})$ whereas that of midazolam group is $21.77 \mathrm{~kg}(+/-4.11$ $\mathrm{kg}$ ) which is not statistically significant. The mean height of children in ketamine group is $114.37 \mathrm{~cm} \mathrm{(+/-}$ $11.17 \mathrm{~cm}$ ) whereas that of midazolam group is 107.93 $\mathrm{cm}(+/-9.96 \mathrm{~cm})$ which is significant. The distribution of sexes in both the groups was as follows: $60 \%$ males and $40 \%$ female children in midazolam group and $56.7 \%$ and $43.3 \%$ females in ketamine group. The distribution of sexes in both the groups was not statistically significant.

Atrial septal defect was the most common procedure undergone with $33.3 \%$ in midazolam group and in $23.3 \%$ in ketamine group followed by ventricular septal defect $30 \%$ in both the groups. The distribution of the type of procedure in both the groups was not statistically significant.

The mean duration of onset of sedation in ketamine group is 21.13 minutes $(+/ 3.78$ mins $)$ whereas it is 15.6 minutes (+/- 2.86 minutes) in midazolam group which is statistically significant. [Table 2]

The response to local anaesthetic infiltration for intravenous cannulation was A response in $3.3 \%$, B 
response in $33.3 \%$ and $\mathrm{C}$ response in $63.3 \%$ of children in ketamine group whereas it is $13.3 \%$ of $\mathrm{B}$ response and $86.7 \%$ of $\mathrm{C}$ response in midazolam group which is statistically significant.

The response to parental separation was B response in $30 \%$ and $70 \%$ of children in ketamine group whereas it was $100 \% \mathrm{C}$ response in midazolam group which is statistically significant. [Table 3]

The response to positioning was $\mathrm{B}$ response in $13.3 \%$ and $\mathrm{C}$ response in $86.7 \%$ of ketamine group whereas it is $100 \% \mathrm{C}$ response in midazolam group which is statistically significant.

The response to local anaesthetic infiltration for femoral vessels catheterization has $\mathrm{B}$ response in $6.7 \%$ and $\mathrm{C}$ response in $93.3 \%$ of ketamine group whereas it is $100 \% \mathrm{C}$ response in midazolam group which is statistically insignificant.

The pulse rates at various intervals of times are shown in the tabular column and it could be found that there is significant difference between the two groups with ketamine group having more of tachycardia than midazolam group which is statistically significant.

The pre induction saturations were comparable in both the groups of ketamine and midazolam. It can be seen from the above table that there is no significant difference between the two groups in pulseoximetry calculated during the study.

There was rise in systolic blood pressure, diastolic blood pressure and MAP in the ketamine group than in the midazolam group which is statistically significant.

Side effects were seen more in ketamine group. [Fig. 1]

Requirement of additional drugs like glycopyrrolate, propofol and supplemental oxygen therapy was seen more with ketamine group.

The mean total time for catheterization study is 108.57 minutes $(+/$ _ 12.44 minutes $)$ in ketamine group whereas it is $100.63(+/-10.35$ minutes $)$ in midazolam group which is statistically significant.

The mean total time spent in recovery room is 53.33 minutes (+/- 10.61 minutes) in ketamine group whereas it is 35.17 minutes (+/- 7.25 minutes) in midazolam group which is statistically significant.

The mean visual analogue scale marked by the parents was $7.27(+/-0.98)$ in the ketamine group when compared to the $8.23(+/-1.65)$ in the midazolam group which is statistically significant. [Fig. 2]

Table 2: Comparison of onset of sedation between ketamine and midazolam group

\begin{tabular}{|c|c|c|c|c|c|c|c|c|c|}
\hline \multirow[t]{3}{*}{ Variables } & \multicolumn{6}{|c|}{ Group } & \multirow{2}{*}{\multicolumn{3}{|c|}{ Unpaired t-test applied }} \\
\hline & \multicolumn{3}{|c|}{ Ketamine } & \multicolumn{3}{|c|}{ Midazolam } & & & \\
\hline & No. & Mean & SD & No. & Mean & SD & t-value & p-value & Difference is- \\
\hline $\begin{array}{l}\text { Observable Onset } \\
\text { of Sedation (mins) }\end{array}$ & 30 & 21.13 & 3.78 & 30 & 15.60 & 2.86 & 6.398 & $2.98 \mathrm{E}-08$ & Significant \\
\hline
\end{tabular}

Table 3: Parental Separation among ketamine and midazolam group

\begin{tabular}{|l|c|c|c|c|}
\hline \multirow{2}{*}{ Parental Separation } & & \multicolumn{2}{c|}{ Group } & \multirow{2}{*}{ Total } \\
\cline { 2 - 4 } & & Ketamine & Midazolam & \\
\hline B & No. & 9 & 0 & $15.0 \%$ \\
\hline C & $\%$ & $30.0 \%$ & $0.0 \%$ & 51 \\
\hline Total & No. & 21 & 30 & $85.0 \%$ \\
\hline & $\%$ & $70.0 \%$ & $100.0 \%$ & 60 \\
\hline Chi-square Tests & No. & 30 & 30 & $100.0 \%$ \\
\hline Pearson Chi-Square & $\%$ & $100.0 \%$ & $100.0 \%$ & Association is- \\
\hline Continuity Correction & Value & df & p-value & Significant \\
\hline
\end{tabular}




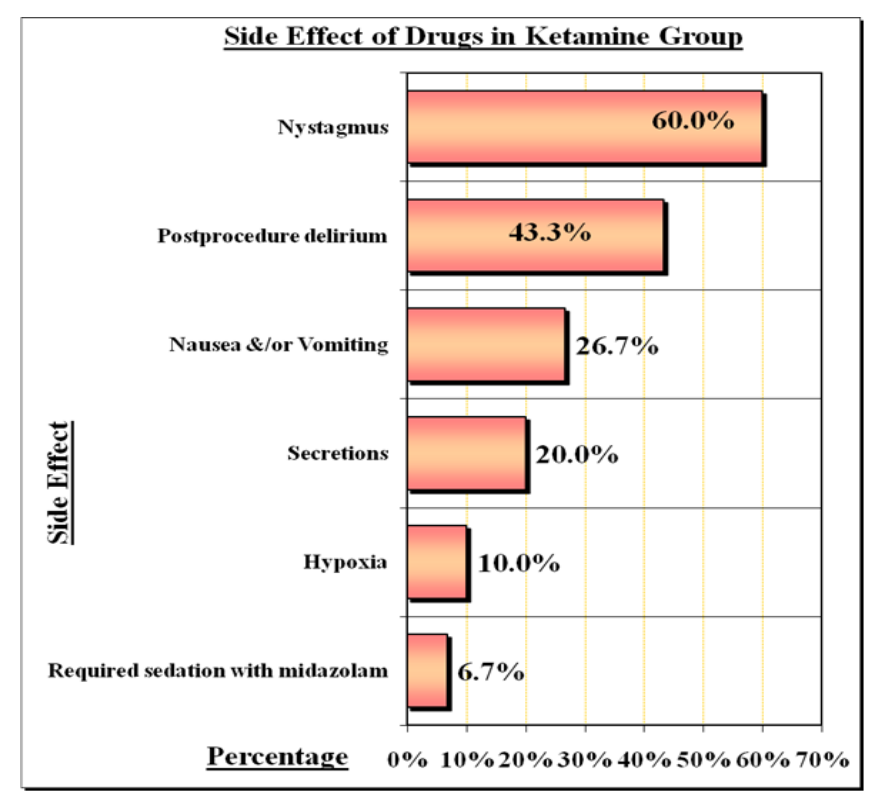

Fig. 1

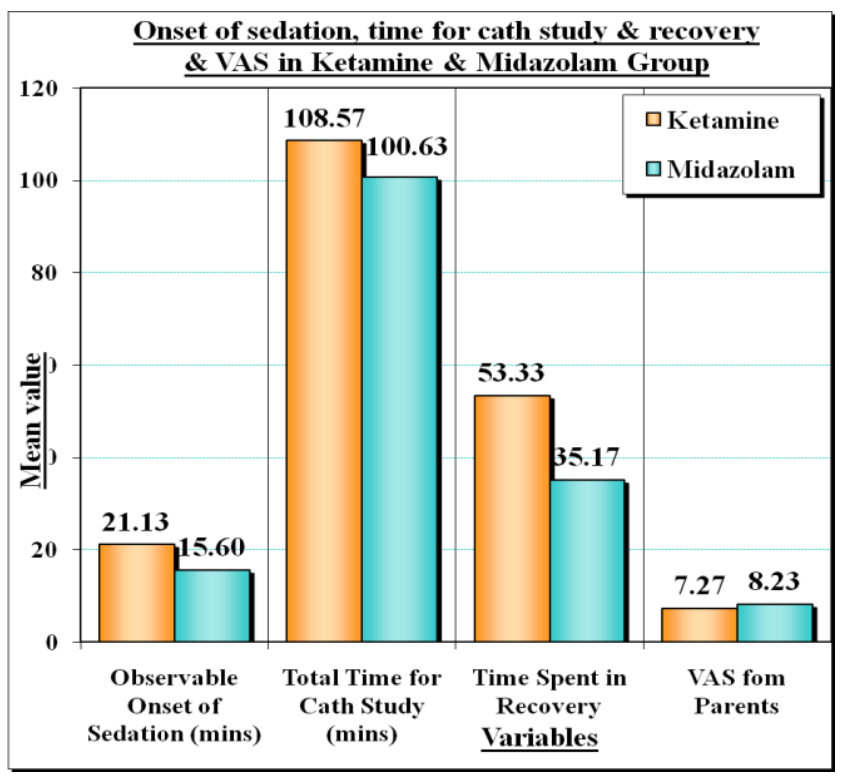

Fig. 2

\section{Discussion}

In the recent years there has been a steady increase in the number of pediatric cardiac patients undergoing cardiac catheterization for diagnostic and therapeutic procedures. Various drugs have been used to provide sedation and pre medication. Despite reassurance by parents, surgeons and anaesthesiologist, a large number of children (40 to $60 \%$ ) still remain anxious peri operatively and an equal number of children suffer from postoperative maladaptive behaviors even weeks after surgical experience. Use of an effective sedative premedicant significantly minimizes the emotional trauma associated with perioperative anxiety and its sequelae. Several sedative drugs administered via different routes have been studied and used as premedicants successfully with minimal risk of adverse effects. Currently, oral midazolam is the most commonly used premedicant in most of the countries followed by oral ketamine.

This study was done to compare the efficacy and safety of orally administered ketamine and midazolam for premedication in children undergoing elective cardiac catheterization procedures in Sir J J Group of hospitals, Grant Medical College, Mumbai.

The demographic values i.e.., the mean age, sex distribution, male female ratio and weight were comparable in both the group and were found to be statistically insignificant.

Before pre medication. The heart rates in both the groups were comparable with $102.33+/-12.09$ beats per minute in ketamine group and with $99.07+/-10.92$ beats 
per minute in midazolam group. The results were statistically insignificant. From 20 minutes onwards till the end of the procedure, there is statistically significant increase in heart rate in ketamine group than in midazolam group. This can be explained by the central stimulation of sympathetic system activation by ketamine leading to increases in heart rate following its administration.

The systolic, diastolic and mean arterial pressures were monitored similar to that of heart rate from the onset of administration of drugs till the discharge of patient from the recovery room.

Before pre medication: The mean arterial pressure before pre medication was $78.13+/-7.97 \mathrm{~mm}$ of $\mathrm{Hg}$ whereas in the midazolam group it was $73.85+/-8.69$ $\mathrm{mm} \mathrm{Hg}$. The results were not statistically significant. After pre medication: The mean arterial pressure after the pre medication significantly rises significantly with ketamine group than midazolam group till the end of the procedure. This as explained earlier may be due to the central sympathetic system activation by the ketamine. Whereas midazolam does not cause stimulation of central sympathetic system and hence there is no increase in mean arterial pressure. This is in accordance with the study conducted by Okluetal. ${ }^{8}$

Before pre medication the mean SPO2 in the ketamine group was $97.9 \%+/-2.16 \%$ and that in midazolam group was $98.2 \%+/-2.27 \%$ which is not statistically significant. After pre medication the mean sPO2 had not changed in a statistically significant way till the end of procedure in either groups except for a fall in saturation at 60 mins 65 mins 160 mins and 170 mins in the ketamine group which required oxygen supplementation.

The mean duration of onset of sedation in ketamine group is 21.13 minutes $\left(+/{ }_{-} 3.78 \mathrm{mins}\right)$ whereas it is 15.6 minutes (+/- 2.86 minutes) in midazolam group which is statistically significant. This signifies that oral midazolam has a earlier onset of sedative action as early as 15 minutes whereas oral ketamine has onset of sedative action at around 20 minutes.

The response to local anaesthetic infiltration for intravenous cannulation was $\mathrm{A}$ response in $3.3 \%, \mathrm{~B}$ response in $33.3 \%$ and $\mathrm{C}$ response in $63.3 \%$ of children in ketamine group whereas it is $13.3 \%$ of $\mathrm{B}$ response and $86.7 \%$ of $\mathrm{C}$ response in midazolam group which is statistically significant. In this study, most of the patients $(86.6 \%)$ showed $\mathrm{C}$ response in midazolam group while rest shows B response, whereas in ketamine group $3.3 \%$ showed A response which is unusal considering the analgesic effects of ketamine and $33.3 \%$ showing $B$ response and rest $63.3 \%$ showing $\mathrm{C}$ response. This is in accordance to the study conducted by Levine MF, et al. ${ }^{9}$

The response to parental separation was B response in $30 \%$ and $70 \%$ of children in ketamine group whereas it was $100 \% \mathrm{C}$ response in midazolam group which is statistically significant. This is in accordance to the study conducted by Levine MF, et al. ${ }^{9}$

These results coincide with the results concluded by other similar studies. Alderson P.J. et al in 1994 found in their study $80 \%$ of the children premedicated with $5 \mathrm{mg} / \mathrm{kg}$ oral ketamine showed satisfactory parentchild separation. ${ }^{10}$

Funk W, Jakob W, Riedl T, Taeger Kin 2000 found in their comparative study ${ }^{11}$ that parent-child separation was satisfactory in $70 \%$ of the children premedicated with $0.5 \mathrm{mg} / \mathrm{kg}$ of oral midazolam whereas only $51 \%$ of the children premedicated with $6 \mathrm{mg} / \mathrm{kg}$ of oral ketamine showed satisfactory parent-child separation.

Another study performed by Cote C.J. et $\mathrm{al}^{12}$ showed that midazolam was effective in producing satisfactory sedation and anxiolysis in $97 \%$ of the children. McMillan C.O. ${ }^{13}$ and colleagues in 1992 found that sedation and anxiolysis scores in the midazolam premedicated groups were excellent in 80$90 \%$ of the children.

The response to positioning was $\mathrm{B}$ response in $13.3 \%$ and $\mathrm{C}$ response in $86.7 \%$ of ketamine group whereas it is $100 \% \mathrm{C}$ response in midazolam group which is statistically significant. Though we could find no studies regarding response to positioning of the patient after sedation in cardiac catheterization study, it could be found from our study that midazolam had resulted in a calm patient with no or minimal response whereas with ketamine there was moderate to minimal response while positioning the patient.

The response to local anaesthetic injection for femoral vessels cannulation were similar with no statistical significance though in ketamine group $6.7 \%$ of patients had moderate response whereas in midazolam group all had minimal or no response indicating the superior efficacy of midazolam over ketamine in providing sedation.

The mean total time for catheterization study is 108.57 minutes (+/_ 12.44 minutes) in ketamine group whereas it is $100.63(+/-10.35$ minutes $)$ in midazolam group which is statistically significant. This cannot be extrapolated to say that oral midazolam results in shorter duration of procedure than oral ketamine for there are many factors affecting the catheterization study including the skills of paediatric cardiologist, the complexity of clinical condition and the nature of the study.

It can be seen that the mean total time spent in recovery is around 18.16 minutes lesser in the midazolam group rather than ketamine group. This has been seen in a study by Cote et al. ${ }^{12}$

P. J. Alderson and J. Lerman in 1994, found out that the time until the children were fit for discharge from the hospital after midazolam was approximately 20 minutes less than after ketamine. ${ }^{10}$

The mean visual analogue scale marked by the parents was $7.27(+/-0.98)$ in the ketamine group when compared to the $8.23(+/-1.65)$ in the midazolam group 
which is statistically significant. This is in accordance with the study by Kain, Z.N. et al in $1998 .{ }^{14}$

In our study adverse effects were seen more in ketamine group, as observed in many studies, Funk W, Jakob found that Vertigo and emesis before induction were significantly more frequent after ketamine premedication $^{11}$ and Younge P. A \& Kendall J. M found that Vomiting was more common with ketamine than in the midazolam group. ${ }^{15}$

McMillan CO et al in 1992 found that the side effects like loss of balance and head control, blurred vision and dysphoric reactions were observed only in the 0.75 and $1.0 \mathrm{mg} \mathrm{kg-1}$ midazolam group. ${ }^{13}$

\section{Conclusion}

From the present study it can be concluded that oral midazolam as a premedication for paediatric cardiac catheterization As it has better hemodynamic stability and sedative effects early onset of sedation, better parental separation, lesser requirement of other drugs, lesser adverse effects, earlier recovery and discharge benefit and better parental satisfaction than oral ketamine.

\section{References}

1. Ellen McCann, Mary, Kain ZN. The management of preoperative anxiety in children: An update in pediatric anesthesia. Anesth and Analg. 2001;93:98-105.

2. Vetter TR. The epidemiology and selective identification of children at risk for preoperative anxiety reactions. Anesth and Analg. 1993;77(1):96-99.

3. Kain ZN, Mayes LC, O Connor TZ, Cicchetti DV. Preoperative anxiety in children: predictors and outcomes. Arch Ped. Adoles Med. 1996;150(12):123845.

4. Kain ZN, Committee on Pediatric Anesthesia. Perioperative psychological issues in children. ASA Newsletteer August 2000;64(8).

5. Hannallah RS, Rosaes JK. Experience with parentsâ€ ${ }^{\mathrm{TM}}$ presence during anesthesia Induction in children. Can J Anesthesia. 1983;30:286-9.
6. Bevan JC, Johnston C. Preoperative parental anxiety predicts behavioral and emotional responses to induction of anesthesia in children. Can J Anesthesia. 1990;37(2):177-82.

7. Raybould D, Bradshaw EG. Premedication for day case surgery: A study of oral midazolam. Anesthesia. 1987;42:591-5

8. Oklu E. Which anesthetic agent alters the hemodynamic status during pediatric catheterization? Comparison of propofol versus ketamine. Journal of cardiothoracic and vascular anesthesia (J. cardiothoracic vasc. anesth.) 2003;17(6):686-690.

9. Levine, M.F., Spahr-Schopfer, I.A., Hartley, E. Can J Anaesth. 1993;40:726.

10. Alderson PJ, Lerman J. Oral premedication for pediatric ambulatory anesthesia: a comparison of midazolam and ketamine. Can J Anesthesia. 1994;41:3,221-26.

11. W Funk, Oral preanaesthetic medication for children: double-blind randomized study. British Journal of Anaesthesia. 84;(3):335-340.

12. Cote C J. Preoperative preparation and premedication: The pediatric patient. British J Anesthesia. 1999;83:1618.

13. McMillan CO, Spahr-Schopfer IA, Skich N. Premedication of children with oral midazolam. Canadian Journal of Anesthesia. 1990;39:545-550.

14. Kain ZN, Mayes LC, and Wang SM. Parental presence during induction of anesthesia versus sedative premedication: Which intervention is more effective Anesthesiology. 1998;89:1147-1156.

15. Younge PA, Kendall JM. Sedation for children requiring wound repair: a randomised controlled double blind comparison of oral midazolam and oral ketamine. Emerg Med J. 2001;18(1):30-3.

How to cite this article: Raja SG, Sarate GS. A comparative study of efficacy and safety of oral ketamine and oral midazolam as premedicant for paediatric cardiac catheterization. Indian $\mathrm{J}$ Clin Anaesth. 2018;5(3):354-360. 